\title{
Unconventional Implant Placement IV. Implant Placement through Impacted Teeth to Avoid Invasive Surgery. Long-term Results of 3 Cases
}

\author{
Davarpanah Mithridade ${ }^{1,2, *}$, Szmukler-Moncler Serge ${ }^{1,3}$, Davarpanah Keyvan ${ }^{1,4}$, \\ Capelle-Ouadah Nedjoua ${ }^{1,5}$, Demurashvili Georgy ${ }^{1,6}$ and Rajzbaum Philippe ${ }^{1,7}$
}

${ }^{1}$ EID research group, Paris, France; ${ }^{2} \mathrm{Head}$, Oral Rehabilitation Centre, American Hospital of Paris, Neuilly sur Seine, France, ${ }^{3}$ Professor ac, Oral Biotechnology Laboratory, Dpt of Surgical Sciences, University of Cagliari, Cagliari, Italy; ${ }^{4}$ Assistant, Dpt of Prosthetics, Bretonneau Hospital, University of Paris 5, Paris, France; ${ }^{5}$ Clinical Monitor, EID research group, Paris, France; ${ }^{6}$ Assistant, Dpt of Prosthetics, Charles Foix Hospital, University of Paris 5, Paris, France; ${ }^{7}$ Fellow, Oral Rehabilitation Centre, American Hospital of Paris, Neuilly sur Seine, France;

\begin{abstract}
This paper presents the long-term data of patients that have been treated with an unconventional implant placement protocol to avoid an invasive surgery when edentulism was caused by an impacted tooth. In 2009, the followup of this unconventional protocol was 2 to 3.5 years; this article documents now the long-term 5- to 8-year follow-up of 3 patients and 5 implants. Over this period of time, implant stability was maintained without complications. This unconventional protocol opens intriguing possibilities; however, more patients with long-term follow-up are warranted before endorsing it in routine application. Nonetheless, it might suggest that there is still room to revisit one of the leading concepts in dental implantology.
\end{abstract}

Keywords: Impacted canine, implant-bone interface, implant-dentine interface, non-invasive surgery, paradigm shift.

\section{INTRODUCTION}

Consider that at the inception of osseointegration, only 2stage surgeries with titanium ancillary were recommended; post-operative radiographs were commended to be performed only at the end of a 3 to 6 -month healing period; occlusal surfaces other than resin like metal or ceramics were barred from use [1]. One can then come to the conclusion that clinical protocols have dramatically changed over time; that paradigms-shifts have been common over the last 35 years. Still, osseointegration is widely ipso facto accepted as 'implant surface should come into contact only with bone', at the exclusion of any remnant tooth material [2].

In 2009, the first 2 clinical papers dealing with implants deliberately placed in contact with dental tissues were published. The related procedures were aiming at dodging invasive surgeries while treating ankylosed [3] or impacted teeth [4]. The follow-up of these prosthetically driven implants placed through dental tissues was ranging 12 to 49 months and 2 to 3.5 years, respectively.

Since then, more cases with impacted teeth have been successfully treated $[5,6]$ and the principle governing these protocols has been extended to inserting implants through residual roots $[7,8]$ or in contact with radicular dentine while seeking to optimize esthetics $[9,10]$. The biological rationale of bringing implants into contact with dental tissues has been extensively addressed elsewhere [3-6]; it was found to get a trustworthy support from available histological data.

*Address correspondence to this author at the 36 rue de Lubeck, F-75116 Paris, France; Tel: + 3315370 36 36;

E-mail: davarpanah@perioimplant.fr
It is pointless to say that these innovative protocols need long-term documentation with large numbers of patients before being accepted in routine use by the clinical community. A large number of cases are not at hand because the present indication involves at best a few patients per year in the hands of every practitioner; getting sizable numbers requires time. Before getting the compulsory numbers, the way to start paving a conceivable route for such an unconventional protocol is to provide long-term documentation even on a small numbers of cases; this would help starting evaluating its long-term fate.

The first few cases with impacted teeth that have been treated with this unconventional implant protocol have been reaching now a follow-up of 5 and more years. The aim of the present paper is, therefore, to report on these first longterm data.

\section{MATERIAL \& METHODS}

\section{Inclusion Criteria and General Requirements}

Conduct of the treatment adhered to the tenets of the latest available Declaration of Helsinki released by the World Medical Association. In addition, to undergo this unconventional treatment, the following conditions had to be met:

1) Patients had to be healthy and able to maintain good hygiene.

2) Implant therapy was indicated.

3) The impacted teeth had to be asymptomatic and free of surrounding pathology. 
4) When treatment planning was discussed with the patient, it was explained that extraction might be complex and invasive; it would require additional augmentation procedures before implant placement. Also, the cortical table might collapse and require an additional bone augmentation procedure before or during implant placement.

5) The patient had to require an alternative option, less invasive and fostering the delivery of the implantsupported prosthesis;

6) A benefit/risk analysis was presented to the patient. Advantage of the protocole was that the patient could avoid an invasive surgery including a bone grafting procedure with a xenogenic material. He/she would be treated according to a standard implant placement procedure without delay after a standard integration period. Another advantage was that the risk of cortical table collapse was circumvented.

The risk of the present protocol was post-operative pain or implant failure after having encroached the impacted tooth. Would it happen, the implant would be immediately removed and the standard extraction procedure of the impacted tooth would follow. The site would then involve bone grafting and healing before placing an implant. Any complication would delay implant placement.

After explanation of the protocol and its deviation from standard care, the patient had to accept the risk of implant failure. In case of implant failure, the conventional treatment with an augmentation procedure was warranted at no additional cost.

7) An informed consent had to be signed.

All patients received standard antibiotic prophylaxis (Augmentin, 2 x 1g/d for 6 days, GlaxoSmithKline, Marly-le-Roi, France).

\section{Evaluation of the Implants}

Implants were evaluated clinically and radiography at the end of the healing period, at 6 months, and then annually. The success criteria according to Cochran et al. [11] were used. Clinical criteria included: 1) absence of clinically detectable mobility, 2) absence of pain or any subjective sensation, 3) absence of peri-implant infection. Radiographic success included: 1) absence of continuous radioluscency around the implant, 2) observation of any abnormal reaction at the bone-implant interface, 3 ) observation of any specific reaction at the root-implant interface, 4) determination of resorption of the remaining root fragment.

\section{Case Presentation and Surgical Procedures of Implant Placement}

\section{Patient \# 1}

A 31-year old female patient attended to rehabilitate her maxillary right canine site. Her temporary canine was lost recently; the site was edentulous because the deciduous canine was impacted. She was wearing a provisional appliance and wished a fixed solution. Radiographic examination showed that the impacted canine was close to the alveolar ridge (Fig. 1a). For esthetic reasons, the patient vigorously refused to consider the orthodontic path prescribed by previous practitioners. The classical surgical approach was then explained; it consisted in removing the impacted canine, grafting the area and placing an implant after 6 months of healing and again waiting for the same amount of time. She was desperate for a shorter and less invasive solution.

To meet the needs of the patient, an alternative noninvasive protocol was then proposed; it was relying on a previous case that has been successful during 6 months until the canines were removed $[4,5]$. It was stressed that would this treatment fail, she would go for the conventional way at no additional cost. The patient accepted to cope with the risk.

A large diameter tapered implant was planned (NT Osseotite, Ø 5 × 15 mm, 3i, Palm Beach Gardens, FL, USA). The impacted site was drilled following the manufacturer recommended drilling sequence, i.e. with the $\varnothing 3.25$ drill (Fig. 1b, c), the $\varnothing 4 \mathrm{~mm}$ and $\varnothing 5 \mathrm{~mm}$. The $\varnothing 6 \mathrm{~mm}$ drill was also used over the coronal half of the osteotomy; the aim was to hope keeping away the dental implant from direct contact with the root of the impacted canine. The coronal portion of the crown was totally removed and the implant was placed (Fig. 1d). Primary stability was achieved at implant seating but the palatal side was left with a bone defect; it was then filled with Bio-Oss ${ }^{\circledR}$ (Geistlich AG, Switzerland), a bone substitute of bovine origin. The gingiva was sutured over the implant.

\section{Patient \# 2}

A 80-year old men attended with a failing mandibular tooth-supported prosthesis. The panoramic radiograph showed that all teeth needed extraction; in addition an impacted premolar was found (Fig. 2a). The impacted premolar was horizontal and the level of impaction was classified as level C [12], i.e. the crown of the impacted tooth was beneath the root apices of the adjacent teeth. Impaction was not associated with a pathological image on the CT scan sections. The patient was seeking a global prosthetic solution in the mandible.

Implant simulation showed that there was no way for the implants to avoid encroaching upon the impacted premolar. It was explained to the patient that the conventional treatment would require extraction of all the remaining teeth, removing invasively the globulous impacted premolar and grafting the created major bone defect. Implants would be placed after a healing period of 6 months. In addition, wearing a temporary prosthesis was strongly dissented for at least 8 weeks in order to protect the grafted sites. The patient asked for a shorter and less invasive alternative.

A non-invasive 2-step solution was therefore discussed. It was proposed to extract all teeth but the canines and place 5 implants; 2 of them would be encroaching upon the impacted premolar in sites WHO \# 32 (ADA \# 23) and \# 34 (ADA \# 21). During the 3 months required to achieve implant stability, the canines would support a provisional bridge. At the $2^{\text {nd }}$-stage surgery, the canines would be extracted and 2 more implants would be placed in the extraction sites. The provisional bridge would be then further prepared to rely on the 5 integrated implants and the 2 newly placed ones. 


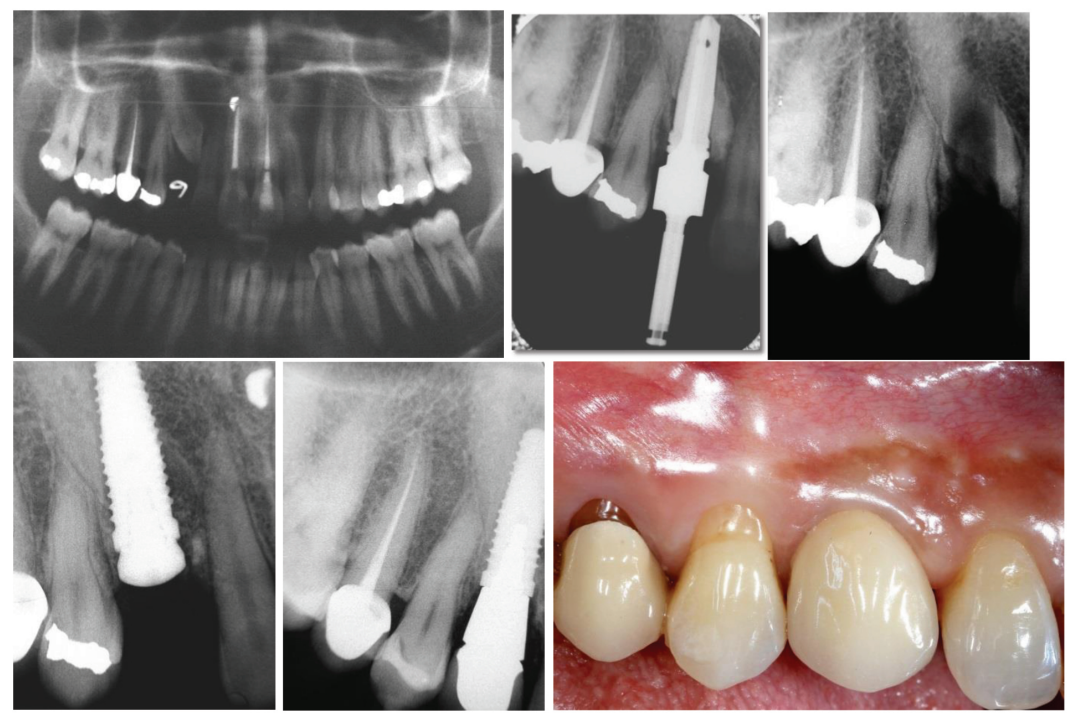

Fig. (1). Patient \# 1. a) Panoramic radiograph with the unerupted canine. b) Radiograph showing the drilling through the impacted canine. c) Radiograph after removing the drill. Reduction in radio-opacity is related to the removal of dental tissue. d) Implant placement after removing the mobile fragment of the crown on the mesial side. e) Periapical radiograph at the 8-year control. f) Vestibular clinical view at the 8-year control. The papilla length is similar to the adjacent natural teeth.

Eventually, 3 out of the 7 implants did encroach upon the impacted premolar; all achieved primary stability. Implant at site WHO \# 34 (ADA \# 21), crossed the premolar root (Fig. 2b), its apical extremity was in contact with bone. At site WHO \# 33 (ADA \# 22), the implant apex was kept within the impacted crown without contacting bone (Fig. 2c). Implant at site WHO \# 32 (ADA \# 23) had its distal side in contact with the cuspidal edge of the crown (Fig. 2d). Implants were Osseotite Certain $\varnothing 4 / 5 \times 13 \mathrm{~mm}$, full Osseotite NT Ø $5 \times 11.5 \mathrm{~mm}$ and $\varnothing 4 \times 13 \mathrm{~mm}$, respectively.

\section{Patient \# 3}

A 85-year old women attended to rehabilitate her atrophic edentulous maxilla. The root of an ankylosed impacted canine was found outcropping the crest on the right side. Bone grafting has been previously performed to receive implants (Fig. 3a). The angulated root canine was occupying a position of strategic importance for implant placement. It was decided to maintain it in order to host an encroaching implant with sufficient primary stability. Implant simulation showed that at least $50 \%$ of the implant surface would be in contact with bone. Nine implants were placed in position WHO \# 11, 12, 13, 14, 16, 17, 21, 25, 26 (ADA \# 2, 3, 5, 6, $7,8,9,13,14)$ and left to heal in a submerged way for 6 months (Fig. 3b).

\section{RESULTS}

\section{Patient \# 1}

No post-operative pain was reported by the patient. The 6-month submerged healing period was uneventful. After checking for implant stability the prosthetic steps were carried out and a single ceramo-metallic crown was delivered. The patient was happy that the edentulous site could be treated in a single non-invasive session, in contrast to the treatment offered by various other practitioners.
At the 8-year control, the implant was clinically stable devoid of any subjective symptoms. Bone has filled the original post-operative defect; platform-shifting has been successful in retaining the crestal bone at its pristine level (Fig. 1e). Radiographically, no abnormal reaction could be observed either at the bone-implant or at the root-implant interface. The remaining root fragment was present.

The pink and white score [13] was 14;. it was reflecting the gingival and bone response to the procedure (Fig. 1f).

\section{Patient \# 2}

Both implant healing procedures were uneventful, no post-operative pain was reported by the patient. The provisional prosthesis was replaced by a final ceramo-metallic bridge.

At the 8-year control, the bridge was clinically stable and implants were successfully fulfilling their function. Attempt to perform a periapical radiograph was unsuccessful because of a pronounced vomiting reflex. Tomodensitometry radiographic examination displayed artefacts at the immediate implants surroundings; however, no specific deleterious feature could be observed (Fig. 2b-e). The crestal bone levels were maintained high, about to reach the level of the neck.

\section{Patient \# 3}

No post-operative pain was reported by the patient. The 6-month healing period was uneventful but perforation of the mucosa by the underneath radicular portion of the canine was recorded at the $2^{\text {nd }}$ stage surgery. The coronal part was flattened with a bur to accommodate the abutment (Fig. 3c, d) and the mesial portion of the root was removed. After another month, the prosthetic steps were undertaken and the final prosthesis was delivered. 

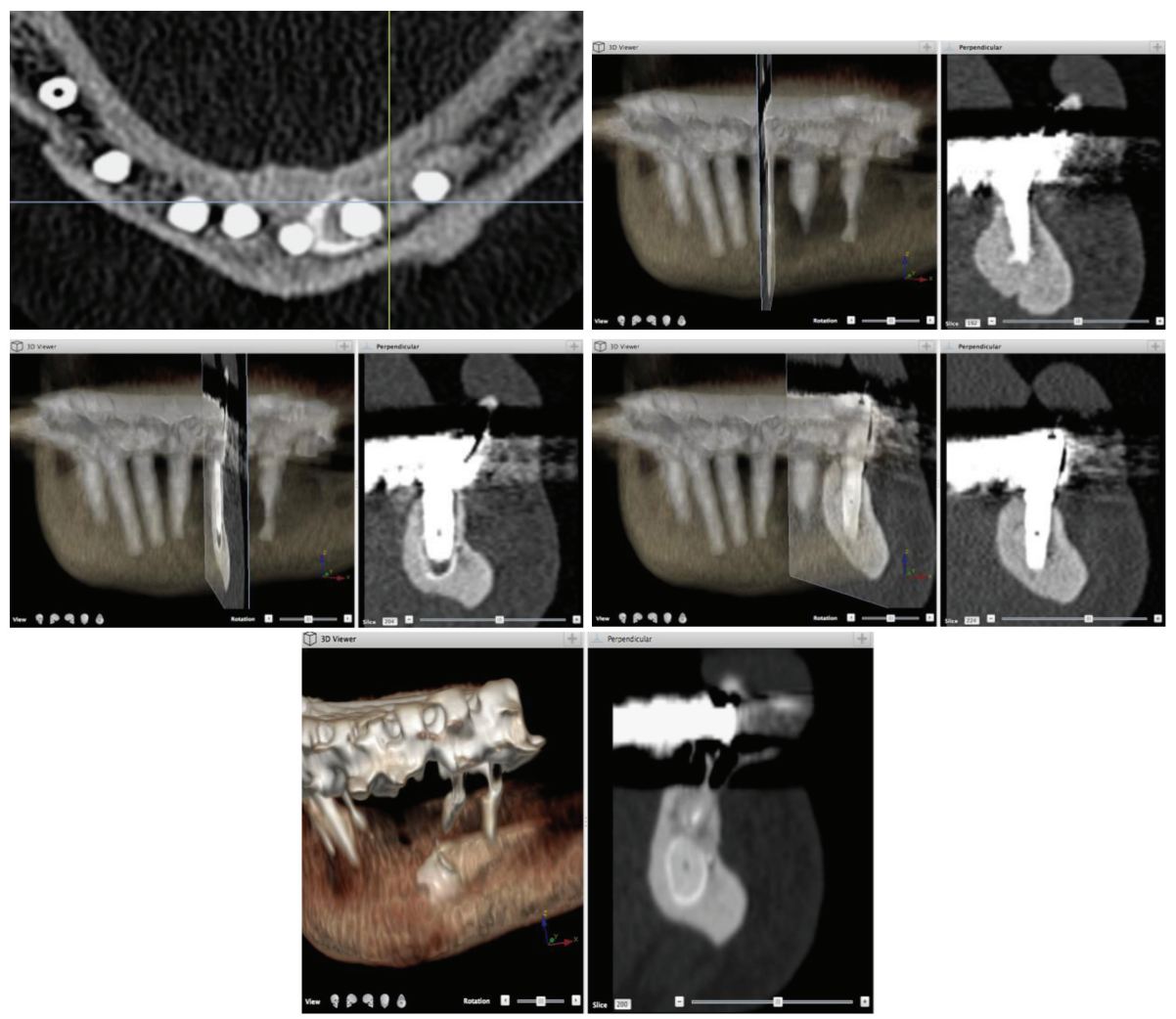

Fig. (2). Patient \# 2. a) Preoperative tomodensitometric examination displaying a 3D reconstruction and a transverse section at the impacted ectopic horizontal premolar. The impacted tooth is in the middle of the mandible just beneath the root of the failing teeth. b) Localisation of implant WHO \# 32 (ADA \# 23) and corresponding transverse section of the tomodensitometric examination at the 8-year control. c) Localisation of implant WHO \# 33 (ADA \# 22) and corresponding transverse section at the 8-year control. d) Localisation of implant WHO \# 34 (ADA \# 21) and corresponding transverse section at the 8-year control. e) Axial section of the tomodensitometric examination at the 8-year follow-up. Note the distinct position of each implant in contact with the various parts of the impacted premolar, the cuspid of the crown, the crown and the root. No specific deleterious radiographic feature could be observed.

At the 5-year control, the prosthesis was stable; the implant placed through the canine did not show any worrying radiographic feature related to the dentine-implant interface (Fig. 3e, f). On the distal side, the dental tissue of the canine retained the level of the most coronal part of the alveolar crest close to its pristine situation (Fig. 3e, f). On the mesial side, bone was close to the level of the neck (Fig. 3e).

\section{DISCUSSION}

This is the first paper reporting on long-term data of implants deliberately placed in contact with dental tissues other than bone with the aim of avoiding an invasive surgery. The reason that led to the developpement of this protocol have been previously explained [3-6]

In all 3 patients, healing was uneventful and postoperative pain was not reported. Implants have been clinically stable and successfully functioning for 5 to 8 years. Five out of six implants have been placed in elderly patients. It is highly probable that the pulp of their impacted teeth was calcified; this might then explain the absence of postoperative pain. However, the same lack of pain at the 31year old patient of this series of cases and other younger patients reported elsewhere [5,6] suggest that absence of pain is the rule rather than the exception. The same absence of pain has been reported when coronoectomy of wisdom teeth close to the alveolar nerve was applied in order to avoid injuring it or to move the impacted tooth away from the nerve $[14,15]$.

This unconventional protocol allowed implementation of implant placement following a conventional surgical procedure. Dodging it would had led the patients to a surgical track of more than one surgery until reaching the standard conditions of implant placement. The treatment would had been then delayed by at least 6 months, probably more.

Several authors reported that orthodontic canine alignment is not as predictable in adults as in teenagers [16-18]. Becker \& Chaushu [19] even stressed that this handling failed in all patients over 30 years. Therefore, in case this unconventional procedure gains recognition by the dental community, it might turn into one of the accepted care options on top of the invasive surgical procedure of tooth removal and further implant placement [20-22].

New interfaces have been created with the implants encroaching upon an impacted tooth; they are the following; 1) an implant-periodontal ligament interface, 2) an implantcement interface, 3) an implant-dentine interface, 4) an implant-pulp interface, 5) an implant-enamel interface.

Some histological data exist to address all these new generated interfaces [2, 9, 23-27] except for the implant enamel 


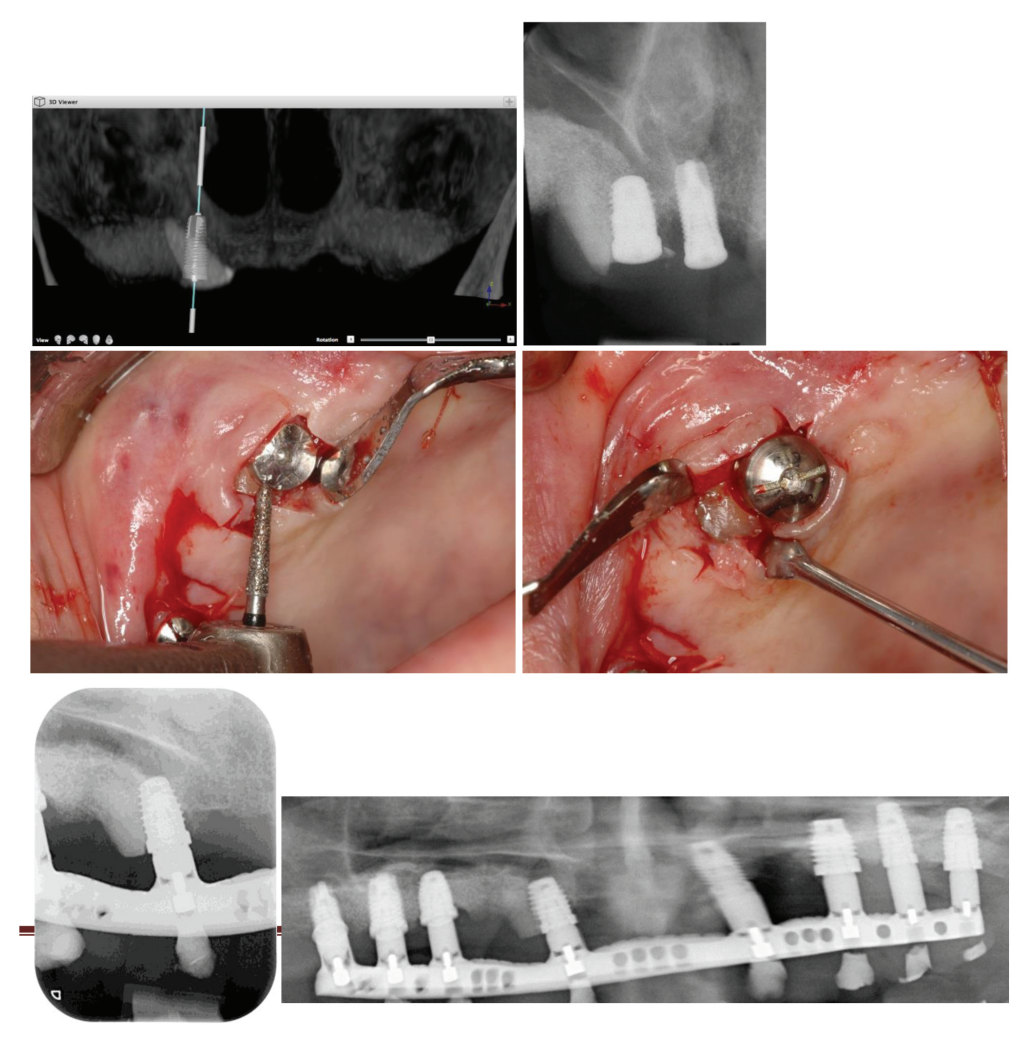

Fig. (3). Patient \# 3. a) Pre-operative panoramic radiograph with the radicular part of the canine. b) Post-operative radiograph of the implant encroaching the root. c) Flattening the distal part of the root of the impacted canine to accommodate the abutment. d) Flattened distal part of the root before suturing over the abutment. e) Periapical radiograph of the implant incroaching upon the canine at the 5-year control. f) Panoramic radiograph of the implant-supported prosthesis at the 5-year control.

interface. Two implants out of the 3 placed in patient \# 2 were encroaching the crown; one of them was only in contact with the cuspid of the crown (Fig. 2e). This means that the enamel-implant interface did not jeopardize implant prognosis. Although these long-term data involve only a limited number of patients and implants, they might serve as a starting substrate to envision positively any new protocol that would require placing implants in contact with dental tissues.

At the beginning, a clinical relevance has been related to 3 distinct indications. Their common goal was to avoid invasive surgeries; they were: placing implants through impacted teeth $[4,7]$, ankylosed teeth $[3,7]$ and residual roots $[7,8]$. But other authors found it applicable to address esthetic issues in order to maintain an optimal support to the marginal gingiva [9] or the papilla [10].

Noteworthy, the present data do not suggest that it should be feasible without consequences to encroach upon a neighbouring vital root in order to place an implant in a narrow space. Because this might lead to either root-canal treatment of the injured dental entity or implant failure [28-31].

Epidemiologic studies have reported that the frequency of impaction of the maxillary canine is low, in the 1-3\% range [32]; indeed, such cases occur once or twice a year in most dental settings. But, then this makes large numbers of patients, in the range of several tens of thousands over the world, and it is worth to work on offering them a possibly relevant patient-friendly surgical protocol.

\section{CONCLUSION}

Placing implants through impacted teeth and generating interfaces other than the implant-bone interface led to uneventful healing; it did not interfere the clinical stability of these implants in the long-term, up to 8 years. More implants are warranted before this protocol can be advocated for routine use but it might open intruiging treatment perspectives. Moreover, it might suggest that there is still room to reevaluate well-anchored paradigms in dental implantology as published elsewhere $[5,6]$.

\section{CONFLICT OF INTEREST}

The authors confirm that this article content has no conflict of interest.

\section{ACKNOWLEDGEMENTS}

The authors are thankful to the efficient help and skills of Aurélie, Vanessa, Alexandra, Vida and Sabrina.

\section{REFERENCES}

[1] Szmukler-Moncler S, Piattelli A, Favero GA, Dubruille JH. Considerations preliminary to the application of early and immediate loading protocols in dental implantology. Clin Oral Implants Res 2000; 11: 12-25.

[2] Gray JL, Vernino AR. The interface between retained roots and dental implants. A histologic study in baboons. J Periodontol 2004; 75: 1102-6.

[3] Davarpanah M, Szmukler-Moncler S. Unconventional implant treatment. I. Implant placement in contact with ankylosed root 
fragments: a series of 5 case reports. Clin Oral Implants Res 2009; 20: 851-6.

[4] Davarpanah M, Szmukler-Moncler S. Unconventional implant treatment. II. Implant placed through impacted teeth. 3 case reports. Int J Periodont Restorat Dent 2009; 29: 405-13.

[5] Davarpanah M, Szmukler-Moncler S, Davarpanah $\mathrm{K}$, et al. Unconventional transradicular implant placement to avoid invasive surgeries: toward a potential paradigm shift. Rev Stomatol Chir Maxillofac 2012; 113: 335-49.

[6] Szmukler-Moncler S, Davarpanah K, Davarpanah M, Rajzbaum P, Capelle-Ouadah N, Demurashvili G. Implants in contact with tissues other than bone. Is there room for a potential paradigm shift? Swiss Dental J 2014; 124: 149-56.

[7] Szmukler-Moncler S, Davarpanah M. Reliability of the rootimplant interface in unconventionally placed implants: an up to 6year follow-up of 23 implants covering 3 distinct clinical applications. Clin Oral Implants Res 2009; 20: 814 (abstract).

[8] Szmukler-Moncler S, Davarpanah M, Davarpanah K, CapelleOuadah N, Demurashvili G, Rajzbaum P. Unconventional implant placement. III. Implant placement encroaching residual roots. A report of 6 cases. Clin Implant Dent Related Res 2014; Jul 17. doi: 10.1111/cid.12256 [Epub ahead of print]

[9] Hürzeler MB, Zuhr O, Schupbach P, Rebele SF, Emmanouilidis N, Fickl S. The socket-shield technique: a proof-of-principle report. J Clin Periodontol 2010; 37: 855-62.

[10] Kan JY, Rungcharassaeng K. Proximal socket shield for interimplant papilla preservation in the esthetic zone. Int J Periodontics Restorative Dent 2013; 33: e24-31.

[11] Cochran DL, Buser D, ten Bruggenkate CM, et al. The use of reduced healing times on ITI implants with a sandblasted and acidetched (SLA) surface: early results from clinical trials on ITI SLA implants. Clin Oral Implants Res 2002; 13: 144-53.

[12] Yavuz MS, Aras MH, Büyükkurt MC, Tozuglu S. Impacted mandibular canines. J Contemp Dent Pract 2007; 8: 78-85.

[13] Fürhauser R, Florescu D, Benesch T, Haas R, Mailath G, Watzek G. Evaluation of soft tissue around single-tooth implant crowns: the pink esthetic score. Clin Oral Implants Res 2005; 16: 639-44.

[14] Pogrel MA, Lee JS, Muff DF. Coronectomy: a technique to protect the inferior alveolar nerve. J Oral Maxillofac Surg 2004; 62: 144752.

[15] Long H, Zhou Y, Liao L, Pyakurel U, Wang Y, Lai W. Coronectomy $v s$. total removal for third molar extraction: a systematic review. J Dent Res 2012; 91: 659-65.

[16] Nordenram A. Impacted maxillary canines: a study of surgically treated patients over 20 years of age. Swed Dent J 1987; 11: 153-8.
[17] McSherry PF. The assessment of and treatment options for the buried maxillary canine. Dental Update 1996; 23: 7-10.

[18] Pitt S, Hamdan A, Rock P. A treatment difficulty index for unerupted maxillary canines. Eur J Orthod 2006 ; 28: 141-4.

[19] Becker A, Chaushu S. Success rate and duration of orthodontic treatment for adult patients with palatally impacted maxillary canines. Am J Orthod Dentofacial Orthop 2003; 124: 509-14.

[20] Mazor Z, Peleg M, Redlich M. Immediate placement of implants in extraction sites of maxillary impacted canines. J Am Dent Assoc 1999; 30: 1767-70.

[21] Cardaropoli D, Debernardi C, Cardaropoli G. Immediate placement of implant into impacted maxillary canine extraction socket. Int $\mathrm{J}$ Periodont Restorat Dent 2007; 27: 71-7.

[22] García B, Boronat A, Larrazabal C, Peñarrocha $M$, Peñarrocha $M$. Immediate implants after the removal of maxillary impacted canines: A clinical series of nine patients. Int J Oral Maxillofac Implants 2009; 24: 348-52.

[23] Buser D, Warrer K, Karring T, Stich H. Titanium implants with a true periodontal ligament: an alternative to osseointegrated implants? Int J Oral Maxillofac Implants 1990; 5: 113-6.

[24] Buser D, Warrer K, Karring T. Formation of a periodontal ligament around titanium implants. J Periodontol 1990; 61: 597-601.

[25] Warrer K, Karring T, Gotfredsen K. Periodontal ligament formation around different types of dental titanium implants. I. The selftapping screw type implant system. J Periodontol 1993; 64: 29-34.

[26] Parlar A, Bosshardt DD, Unsal B, Cetiner D, Haytaç C, Lang NP. New formation of periodontal tissues around titanium implants in a novel dentin chamber model. Clin Oral Implants Res 2005; 16: 259-67.

[27] Schwarz F, Mihatovic I, Golubovic V, Becker J. Dentointegration of a titanium implant: a case report. Oral Maxillofac Surg 2013; 17: 235-41.

[28] Margelos JT, Verdelis KG. Irreversible pulpal damage of teeth adjacent to recently placed osseointegrated implants. J Endo 1995; 21: 479-82.

[29] Sussman HI. Tooth devitalization via implant placement: a case report. Periodontal Clin Investig 1998; 20: 22-4.

[30] Kim SG. Implant-related damage to an adjacent tooth: a case report. Implant Dent 2000; 9: 278-80.

[31] Yoon WJ, Kim SG, Jeong MA, Oh JS, You JS. Prognosis and evaluation of tooth damage caused by implant fixtures. J Korean Assoc Oral Maxillofac Surg 2013; 39: 144-7.

[32] Cooke J, Wang HL. Canine impactions: Incidence and management. Int J Periodontics Restorative Dent 2006; 26: 483-91.

\begin{tabular}{lcc}
\hline Received: October 10, 2014 & Revised: November 29, 2014 & Accepted: December 02, 2014 \\
(C) Mithridade et al:; Licensee Bentham Open. & &
\end{tabular}

(1) et al.; Licensee Bentham Open.

This is an open access article licensed under the terms of the Creative Commons Attribution Non-Commercial License (http://creativecommons.org/licenses/by-nc/3.0/) which permits unrestricted, non-commercial use, distribution and reproduction in any medium, provided the work is properly cited. 